\title{
Expression and distribution of SP and its NK1 receptor in the brain-gut axis in neonatal maternally separated rat model with visceral hypersensitivity
}

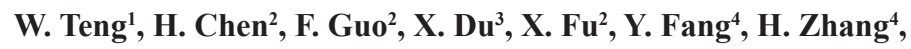 \\ M. Fang ${ }^{3}$ and M. Ding ${ }^{2}$ \\ ${ }^{1}$ Endoscopy Center, Jinhua Hospital of Zhejiang University, Jinhua, China \\ ${ }^{2}$ Department of Medical Sciences, Jinhua Polytechnic, Jinhua, China \\ ${ }^{3}$ Institute of Neuroscience, Zhejiang University School of Medicine, \\ Hangzhou, China \\ ${ }^{4}$ JinHua Center of Laboratory Animals, Jinhua, China \\ Corresponding authors: M. Ding / M. Fang \\ E-mail: dingmx@sina.com / fangmaro@zju.edu.cn
}

Genet. Mol. Res. 15 (3): gmr.15038999

Received July 21, 2016

Accepted August 1, 2016

Published August 30, 2016

DOI http://dx.doi.org/10.4238/gmr.15038999

Copyright (C) 2016 The Authors. This is an open-access article distributed under the terms of the Creative Commons Attribution ShareAlike (CC BY-SA) 4.0 License.

\begin{abstract}
Neurokinin-1 receptor (NK1R) is a high affinity Substance $\mathrm{P}(\mathrm{SP})$ receptor and plays a key role in visceral hypersensitivity in irritable bowel syndrome (IBS). Early life stress is a significant risk factor in IBS. The aim of the present study was to investigate the influence of neonatal maternal separation on the expression and distribution of SP and its receptor along the brain-gut axis in a neonatal maternally separated rat model with visceral hypersensitivity. Male neonatal Sprague-Dawley rats, 2-21-day old, were randomly distributed into maternal separation groups of $3 \mathrm{~h}$ daily maternal separation (MS) or
\end{abstract}

Genetics and Molecular Research 15 (3): gmr.15038999 
non-handling $(\mathrm{NH})$. These rats underwent colorectal balloon distention (CRD) upon reaching adulthood. Immunofluorescence was used to examine the distal colon, lumbosacral spinal cord, and the brainstem to semi-quantitatively determine SP and NK1R expression before and after CRD. The following features were assessed: percentage SPpositive area in colonic muscle layer, the number of NK1R-positive myenteric plexus, SP-positive area and NK1-positivity score in the dorsal horn and the brainstem. Neither of these was altered in the MS and $\mathrm{NH}$ groups before or after CRD. These results suggest that the SP system might play little role in the development of visceral hyperalgesia in the neonatal maternal separation rat model.

Key words: Irritable bowel syndrome; Neonatal maternal separation; Substance P; Neurokinin-1 receptor; Brain-gut axis; Rat

\section{INTRODUCTION}

The gastrointestinal tract is modulated by the central and enteric nervous systems, which establish a mutual communication between the brain and the gut ("brain-gut axis") (Carabotti et al., 2015). The digestive function of organism is optimized by brain-gut signaling regulating motility, secretion, immune function, and blood flow. The etiology of irritable bowel syndrome (IBS) is still unknown, but the current leading hypothesis concerns visceral hypersensitivity. It has been demonstrated that substance P (SP) and its neurokinin-1 receptor (NK1R) play a significant role in nociceptive perception that contributes to visceral hypersensitivity (Ma et al., 2009). SP, a neuropeptide, contains 11 amino acids, belongs to tachykinin family of peptides, and is an excitatory neurotransmitter in the bowel (Lecci et al., 2006). It has been identified in the mammalian nerve tissues, including the brain, spinal cord, and the enteric nervous system of the gut (Lecci et al., 2006; Darmani et al., 2008). SP stimulates nerves and triggers a nociceptive pain sensation via sensory nerves to enhance the motility of the gut. In central nerve system, visceral sensations are transmitted from the gut via afferent nerves, travelling through the spinal cord to the brain where their painful characteristics are perceived. It has been recently reported that an abdominal response caused by intrathecal administration of an NK1R antagonist during colorectal distension (CRD), induced by repeated stress, triggered NK1R up-regulation in the dorsal horn (Bradesi et al., 2006). When inflammation or injury occurred in the gastrointestinal tract, peripheral nerve endings released SP, which directly activated NK1R of the nerve terminals or trigger mast cells eliciting their deregulation (Wang et al., 2014). Direct stimulation of muscle cells and enteric neural circuits, mainly by NK1R-SP interaction, simultaneously stimulates and inhibits bowel motility (Scheurer et al., 1994). On the other hand, activated mast cells continuously discharge several inflammatory factors, which leads to tissue edema and neurogenic inflammation mediated by anapetia, C-type afferent terminals, and others factors. An in-depth exploration of visceral hypersensitivity mechanisms should be based on a successfully established animal model of visceral hyperalgesia that mimics IBS in human. A widely adopted model of early life stress is a newborn maternally separated rat model where the separation results in irrevocable changes in the spinal cord and brain (Coutinho et al., 2002; O'Malley et al., 2011). It has been proposed that maternal separation (MS) of newborn rats can be used as an effective

Genetics and Molecular Research 15 (3): gmr.15038999 
animal model of visceral hyperalgesia (Ren et al., 2007; Tjong et al., 2011). Consequently, the present study aimed to investigate whether SP and NK1R alterations supervened the visceral hyperalgesia after MS in the rat model. Several indices, including percentage SP-positive area in the colonic muscle layer, the number of NK1R-positive myenteric plexus SP-positive area and NK1-positivity scores in the dorsal horn and brainstem, were examined. The expression as well as distribution of SP and NK1R in the colon, spinal cord, and the brainstem will facilitate better understanding of neuromechanisms underlying the transmission of visceral hypersensitivity, and will provide rationale for the IBS therapy.

\section{MATERIAL AND METHODS}

\section{Setting up the rat model}

One-day-old postnatal Sprague-Dawley rats were supplied by the Laboratory Animal Center of Zhejiang University (Hangzhou, China). We chose male pups to eliminate the impact of estrogens and hormonal cycle on the neurochemical and sensory response stimulation of the bowel. Male pups $(\mathrm{N}=64)$ were randomly divided into two groups, MS group and nonhandling $(\mathrm{NH})$ group, according to rearing conditions $(\mathrm{N}=32$ per group). At 2-21-day-old, the litters underwent 3-h daily MS (Coutinho et al., 2002). At $0800 \mathrm{~h}$, the litters were shifted away from the dams, and were placed in an isolation cage for $3 \mathrm{~h}$, as a group, in an adjacent thermoregulated room. Following this, they were transferred back to the maternity cage. Additional pups were kept as a group in a standard cage with a housed dam, under standard conditions. All pups were kept under standardized conditions with sterilized water and pellet food, with $12 \mathrm{~h}$ light-dark cycle. Litters were weaned on day 22 and divided randomly into cages on day 30 (four littermates per cage). Other treatments were performed on day 60 (weighing; average weight: $280-320 \mathrm{~g}$ ). Animals were treated following the guidelines of the Laboratory Animal Center of Zhejiang University. All experimental procedures were approved by the Animal Research Committee of Zhejiang University School of Medicine. Behavioral tests were carried out during the light phase.

\section{Colorectal balloon distention}

Upon reaching adulthood on day 60 , all animals from the two treatment groups were subjected to CRD. First, animals were anesthetized by $7 \%$ chloral hydrate intraperitoneal injection. Balloons were made from 6-cm long latex glove fingers, tied to a balloon dilator, linked by a Y-connector to a syringe pump and a sphygmomanometer (Stam et al., 2002; Ren et al., 2007). Balloon catheter was inserted $1 \mathrm{~cm}$ from the anus and fixed to the distal edge of the anus with a base end of tape. The rats were allowed to wake up and recover for $30 \mathrm{~min}$ on an elevated Plexiglas platform and the conscious animals then underwent 20 rapid phasic CRDs at $60 \mathrm{mmHg}$ with $20 \mathrm{~s}$ inflation followed by 4 min deflation. The duration of CRD of each rat was strictly time-controlled.

\section{Tissue preparation}

Following anesthesia, the animals were quickly perfused with $0.9 \%$ normal saline $(100 \mathrm{~mL})$ and fixed by $4 \%$ paraformaldehyde $(\mathrm{PA} ; 300 \mathrm{~mL})$. The distal colon $(6-7 \mathrm{~cm}$ from

Genetics and Molecular Research 15 (3): gmr.15038999 
the anus), lumbosacral spinal cord (L6-S1), and brainstem were dissected, post-fixed in $4 \%$ PA overnight, following dehydration in $30 \%$ sucrose for $48 \mathrm{~h}$ at $4{ }^{\circ} \mathrm{C}$. After freezing in OCT compound, the embedded tissues were cut into $15-\mu \mathrm{m}$ (colon) or $40-\mu \mathrm{m}$ (spinal cord and brainstem) thick sections for analyses. Colon tissue section was stained with hematoxylin and eosin. The colon, lumbosacral cord (especially the dorsal horn), and brainstem were used for the following reasons. 1) It has been determined that SP and NK1Rs are distributed in the circular and longitudinal smooth muscle layers and in myenteric plexus neurons (Renzi et al., 2000; Boutaghou-Cherid et al., 2006). 2) SP and NK1Rs were here detected in the lumbosacral spinal cord, because the afferent impulses of pain in the thoracolumbar spine segment are unclear. Furthermore, the distal colon and rectum convey afferent stimuli mainly to the lumbosacral spinal cord along the pelvic nerve (Kyloh et al., 2011). 3) Dorsal horn of the spinal cord is a key area of sensitivity modulation. Neurons of the dorsal horn may be stimulated to increase their sensitivity by NK1 or N-methyl-D-aspartate receptor (NMDAR) pathway (Ogawa et al., 2007). NK1R and NMDAR are stimulated by SP and excitatory amino acids, respectively. SP can directly activate dorsal spinal neurons and further influence visceral sensation (Lu et al., 2005). 4) MS causes irreversible changes in the CNS, also in central circuits, which mediate autonomic and pain modulatory responses. It has been determined that brain regions, such as the cingulated cortex, thalamus, hypothalamus, periaqueductal gray (PAG), and dorsal raphe nucleus (DRN), are involved in pain generation and modulation (Chung et al., 2007; Yin et al., 2014).

\section{Immunohistochemistry}

Sections of the colon, spinal cord (L6-S1), and brainstem (midbrain) were rinsed $3 \times 30$ $\min$ in $0.01 \mathrm{M}$ PBS. After preincubation at $37^{\circ} \mathrm{C}$ for $60 \mathrm{~min}$ in $0.01 \mathrm{M}$ PBS with $10 \%$ normal goat serum (NGS) and $0.3 \%$ Triton $\mathrm{X}-10$, the sections were incubated overnight at $4{ }^{\circ} \mathrm{C}$ with mouse anti-SP mono-antibodies (1:500; Abcam, Cambridge, MA, USA) or rabbit anti-NK1R polyclonal antibodies (1:200; Sigma, St. Louis, MO, USA) in 0.01 M PBS containing 5\% NGS and $0.3 \%$ Triton $\mathrm{X}-100$. Rinsed sections were incubated with secondary antibodies at $37^{\circ} \mathrm{C}$ for $60 \mathrm{~min}$, either with goat anti-mouse IgG conjugated with fluorescein isothiocyanate (FITC; 1:50; Sigma, St. Louis, MO, USA) or with goat anti-rabbit IgG conjugated with tetraethyl rhodamine isothiocyanate (TRITC; 1:200; Sigma, St. Louis, MO, USA). The preparations were then mounted using antifade Gel/Mount aqueous mounting medium (Southern Biotech, Birmingham, AL, USA), air-dried, dehydrated, cleared, and coverslipped for microscopic analysis.

\section{Image analysis}

Among the prepared of achieved tissues (colon, spinal cord, and brainstem) were checked carefully under Nikon fluorescence photomicroscope (Nikon, Tokyo, Japan) and images were captured with high-definition cooled color digital camera (DXM1200C, Nikon, Tokyo, Japan). Metamorph 4.0 software (Universal Imaging) was used to analyze the images, and the measurement was automatic. The latter resulted in colored overlay that marked which pixels in the image to be measured. After thresholding, defined "objects" in the images were measured and separated from the background. These procedures determine whether a pixel comprises an unmeasured object or only part of the pixels according to background intensity. All analyses were performed by an analyst blinded to group definitions and treatments.

Genetics and Molecular Research 15 (3): gmr.15038999 
In the colon samples, greenish dots corresponded to SP-positive areas, when FITC in FITC-antibody conjugates was excited by $470 \mathrm{~nm}$ light, and the emitted light was detected at $520 \mathrm{~nm}$. In the spinal cord and brainstem, SP- and NK1R-positive areas were stained green and red, respectively. The following SP- and NK1R-positive areas were measured: tunica muscularis coli and stratum submucosum, colonic myenteric nerve plexus, dorsal horn of the spinal cord (L6-S1), PAG, and DRN of the brainstem. Five fields of view from each section were randomly selected to obtain digitalized images at different magnifications. Image analysis methods were different for SP and NK1R. SP/NKIR-active areas were transformed by the image analysis software to exact pixel number per area, in proportion to the total area. Expression-negative tissue was excluded from analyses. SP-positive area percentage in the colon (the percentage of SP-positive areas compared with tunica muscularis coli areas) was calculated as the size of SP-positive areas within the integral tunica muscularis coli area, in the dorsal horn, and in PAG and DRN of the brainstem. SP-positive area was calculated as $\mu \mathrm{m}^{2} /$ bilateral dorsal horn area $\left(\mathrm{mm}^{2}\right)$. Similarly, percentage SP-positive area in PAG and DRN was calculated as $\mu \mathrm{m}^{2} /$ midbrain $\left(\mathrm{mm}^{2}\right)$ (Inagaki et al., 1982).

For NK1R, the number of NK1R-positive myenteric nerve plexus areas was counted under a 20X objective. Semi-quantitative score was used to analyze the expression and distribution of NK1R in the dorsal horn, and PAG and DRN of the brainstem. Noxious CRD induces the expression of transmitters in the lumbosacral spinal cord, mainly in lamina I, III-V, and X (Kozlowski et al., 2000). Thus, the distribution range of NK1R immunoreaction was measured using semi-quantitation scores according to the number of immuno-positive regions. The scores were as follows: 1, one immuno-reactive region; 2, two immuno-reactive regions; 3 , three immuno-reactive regions; $4,3<$ immuno-reactive regions.

\section{Data analysis}

All data were analyzed by SPSS 22.0 (SPSS, Chicago, IL, USA). Values are reported as the means $\pm \mathrm{SD}$. The comparisons of NK1R and SP expression between two groups were made by Mann-Whitney U test, as appropriate. Comparisons of other indices were done using one-way ANOVA. In all statistical tests, probability value $\mathrm{P}<0.05$ was considered significant.

\section{RESULTS}

\section{Histological changes in the colon}

Histological sections of the rat colons from each group were analyzed. No mucin depletion or increase in mucosal lymphocytes was observed, indicating no structural abnormalities. Histological analysis of the colon showed no colonic inflammation in any group.

\section{SP expression and distribution of SP immuno-positivity in the colon}

In the rat colon, SP immunoreactivity was mainly detected in tunica muscularis coli (Figure 1A). SP-positive nerve fibers were also observed in the circular muscle layer and in myenteric nerve plexus. Moreover, the data indicated that low SP expression was sporadically distributed in stratum submucosum in both, control and maternally-deprived rats. Percentage SP-positive area decreased after CRD. However, SP distribution and expression intensity were

Genetics and Molecular Research 15 (3): gmr.15038999 
not significantly different between the MS and $\mathrm{NH}$ groups, before or after $\mathrm{CRD}$, according to quantitative analyses (Table 1 and Figure 1B).
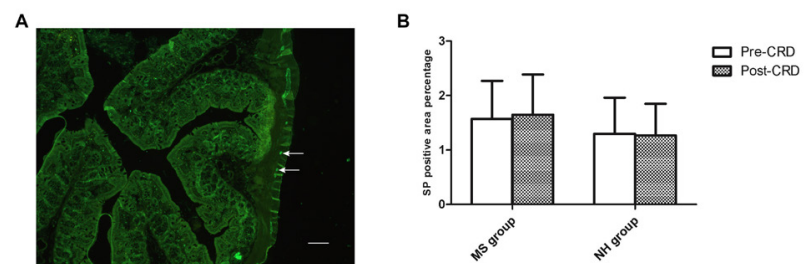

Figure 1. SP expression and distribution in the colon. A. Distribution of SP immunoreactivity in tunica muscularis coli and stratum submucosum. The greenish immunofluorescent dots represent SP-positivity. Scale bar, $20 \mu \mathrm{m}$. B. A decreasing tendency in percentage SP-positive area between MS and NH groups was observed, pre-CRD or post$\mathrm{CRD}$, but a statistically significant difference was not attained $(\mathrm{P}>0.05)$.

Table 1. SP positive area percentage in tunica muscularis coli of the MS and NH groups.

\begin{tabular}{l|c|c}
\hline \multirow{2}{*}{ Group } & \multicolumn{2}{|c}{ SP positive area percentage } \\
\cline { 2 - 3 } & Before CRD & After CRD \\
\hline MS group ( $=16)$ & $1.57 \pm 0.70$ & $1.65 \pm 0.74$ \\
\hline NH group $(\mathrm{N}=16)$ & $1.30 \pm 0.66$ & $1.27 \pm 0.58$ \\
\hline P value & 0.27 & 0.12 \\
\hline
\end{tabular}

NK1R expression and distribution of NK1R immuno-positivity in the colon.

NK1R immunoreactivity in the colon was located predominantly in the myenteric nerve plexus (Figure 2A). Significant differences in the number of NK1R-positive myenteric plexus in both MS and NH rats, before or after CRD, were not detected (Table 2 and Figure 2B).
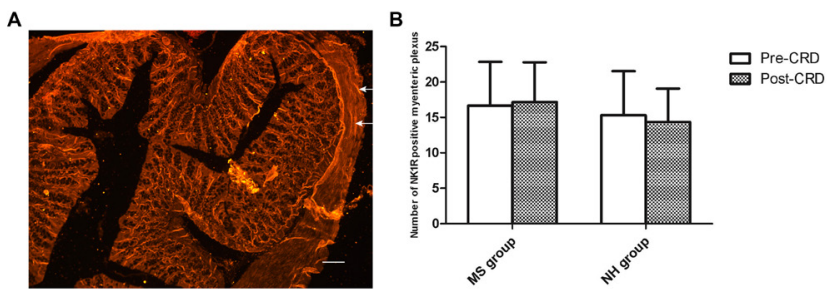

Figure 2. NK1R expression and distribution in the colon. A. Distribution of NK1R immunoreactivity in colonic myenteric nerve plexus. The reddish immunofluorescent dots represent NK1R-positivity. Scale bar, $20 \mu \mathrm{m}$. B. A statistically significant difference in the number of NK1R-positive myenteric plexus between MS group and $\mathrm{NH}$ group, pre-CRD or post-CRD, was not attained $(\mathrm{P}>0.05)$.

Table 2. Number of NK1R positive myenteric plexus in both MS and NH rats.

\begin{tabular}{l|c|c}
\hline \multirow{2}{*}{ Group } & \multicolumn{2}{|c}{ Number of NK1R positive myenteric plexus } \\
\cline { 2 - 3 } & Before CRD & After CRD \\
\hline MS group $(\mathrm{N}=16)$ & $16.65 \pm 6.20$ & $17.16 \pm 5.62$ \\
\hline $\mathrm{NH}$ group $(\mathrm{N}=16)$ & $15.31 \pm 6.22$ & $14.37 \pm 4.70$ \\
\hline P value & 0.55 & 0.14 \\
\hline
\end{tabular}

SP expression and distribution of SP immuno-positivity in the dorsal horn of spinal cord.

Genetics and Molecular Research 15 (3): gmr.15038999 
SP immuno-reactive neurons and fibers were located in the superficial layer of dorsal horn (Figure 3A), but no significant differences were observed in SP-positive areas in the dorsal horn of spinal cord (L6-S1) between MS and NH rats, before or after CRD (Table 3 and Figure 3B).
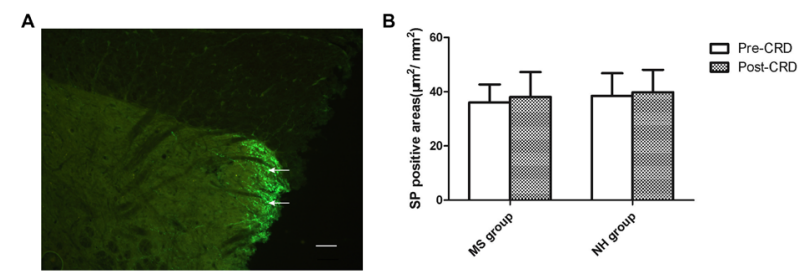

Figure 3. SP expression and distribution of SP in the dorsal horn of spinal cord. A. Expression and distribution of SP immunoreactivity in the dorsal horn of spinal cord. The greenish dots represent SP-reactivity. Scale bar, $40 \mu \mathrm{m}$. B. SP-positive area in the dorsal horn was similar before and after CRD $(\mathrm{P}>0.05)$.

Table 3. SP positive areas in dorsal horn of spinal cord in both MS and NH rats.

\begin{tabular}{l|c|c}
\hline \multirow{2}{*}{ Group } & \multicolumn{2}{|c}{ Substance P positive areas $\left(\mu \mathrm{m}^{2} / \mathrm{mm}^{2}\right)$} \\
\cline { 2 - 3 } & Before CRD & After CRD \\
\hline MS group $(\mathrm{N}=16)$ & $36.01 \pm 6.68$ & $38.07 \pm 9.21$ \\
\hline NH group $(\mathrm{N}=16)$ & $38.47 \pm 8.33$ & $39.85 \pm 8.29$ \\
\hline P value & 0.37 & 0.57 \\
\hline
\end{tabular}

NK1R expression and distribution of NK1R immuno-positivity in the dorsal horn of spinal cord.

Within the dorsal horn of spinal cord, NK1R immunoreactivity was extensive and mainly localized in the superficial layer. NK1R immuno-reactive neurons and neuritis were detected in the deep layer of dorsal horn as well as in the vicinity of central canal (Figure 4A). We observed an increasing tendency of the NK1R-positivity score of the dorsal horn in MS group, but the difference between MS group and NH group was not statistically significant (P $>0.05)$. The expression and distribution of NK1R in MS groups were not altered compared with NH groups before CRD and after repetitive CRD (Table 4 and Figure 4B).
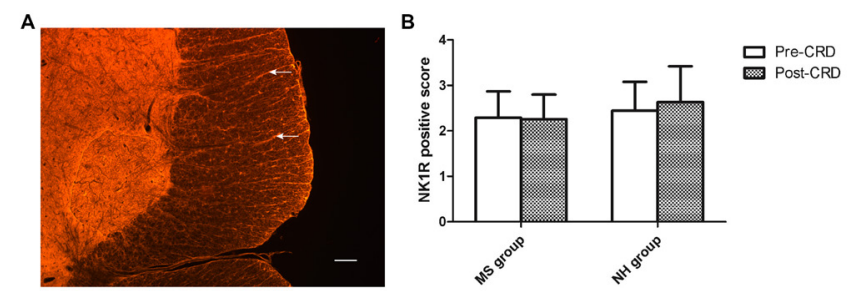

Figure 4. NK1R expression and distribution in the dorsal horn of spinal cord. A. Expression and distribution of NK1R immunoreactivity in the dorsal horn of spinal cord. The reddish dots represent NK1R reactivity. Scale bar, $40 \mu \mathrm{m}$. B. NK1-positivity score in the dorsal horn remained unchanged regardless of CRD (P>0.05).

Table 4. NK1R positive scores in dorsal horn of spinal cord in both MS and NH rats.

\begin{tabular}{l|c|c}
\hline \multirow{2}{*}{ Group } & \multicolumn{2}{|c}{ NK1R positive score } \\
\cline { 2 - 3 } & before CRD & after CRD \\
\hline MS group $(\mathrm{N}=16)$ & $2.29 \pm 0.58$ & $2.26 \pm 0.54$ \\
\hline NH group $(\mathrm{N}=16)$ & $2.44 \pm 0.64$ & $2.63 \pm 0.79$ \\
\hline P value & 0.52 & 0.15 \\
\hline
\end{tabular}

SP expression and distribution of SP immuno-positivity in the brainstem.

Genetics and Molecular Research 15 (3): gmr.15038999 
Some SP-positive neurons were detected in PAG and DRN (Figure 5A), but no significant differences were observed in SP-positive areas in the midbrain (L6-S1) between MS and NH rats, before or after CRD (Table 5 and Figure 5B).
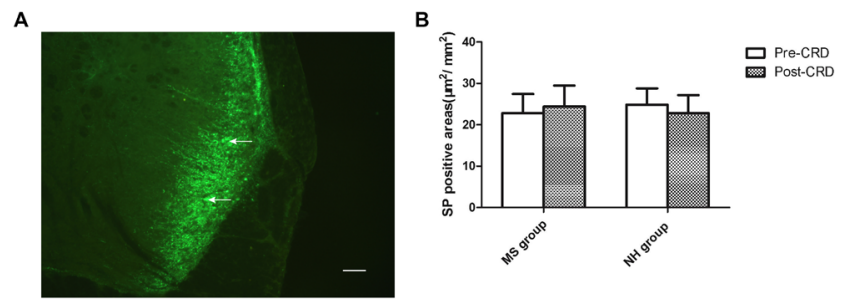

Figure 5. SP expression and distribution in the brainstem. A. Expression and distribution of SP immunoreactivity in the midbrain. The greenish dots represent SP reactivity. Scale bar, $40 \mu \mathrm{m}$. B. SP-positive area in the midbrain was similar before and after CRD $(\mathrm{P}>0.05)$.

Table 5. SP positive areas in midbrain in both MS and NH rats.

\begin{tabular}{l|c|c}
\hline \multirow{2}{*}{ Group } & \multicolumn{2}{|c}{ Substance P positive areas $\left(\mu \mathrm{m}^{2} / \mathrm{mm}^{2}\right)$} \\
\cline { 2 - 3 } & Before CRD & After CRD \\
\hline MS group $(\mathrm{N}=16)$ & $22.79 \pm 4.64$ & $24.43 \pm 5.05$ \\
\hline NH group $(\mathrm{N}=16)$ & $24.84 \pm 3.94$ & $22.78 \pm 4.38$ \\
\hline P value & 0.19 & 0.33 \\
\hline
\end{tabular}

NK1R expression and distribution of NK1R immuno-positivity in the brainstem.

NK1R immunoreactivity was observed in cell bodies and processes throughout the PAG and DRN regions (Figure 6A). We detected an increasing tendency of the NK1R-positive score of PAG and DRN in MS group, but a statistically significant difference was not noted $(\mathrm{P}>0.05)$. The expression and distribution of NK1R in MS groups showed no remarkable change compared with NH groups (Table 6 and Figure 6B).
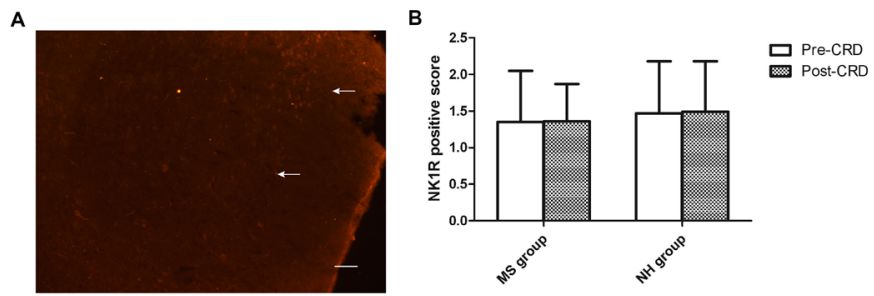

Figure 6. NK1R expression and distribution in the brainstem. A. Expression and distribution of NK1R immunoreactivity in the midbrain. The reddish dots represent NK1R reactivity. Scale bar, $40 \mu \mathrm{m}$. B. NK1-positivity score in the midbrain before and after CRD remained unchanged $(\mathrm{P}>0.05)$.

Table 6. NK1R positive scores in midbrain in both MS and NH rats.

\begin{tabular}{l|c|c}
\hline \multirow{2}{*}{ Group } & \multicolumn{2}{|c}{ NK1R positive score } \\
\cline { 2 - 3 } & Before CRD & After CRD \\
\hline MS group $(\mathrm{N}=16)$ & $1.35 \pm 0.70$ & $1.36 \pm 0.51$ \\
\hline NH group $(\mathrm{N}=16)$ & $1.47 \pm 0.71$ & $1.49 \pm 0.69$ \\
\hline P value & 0.61 & 0.57 \\
\hline
\end{tabular}

Genetics and Molecular Research 15 (3): gmr.15038999 


\section{DISCUSSION}

In IBS patients, the genesis of visceral hypersensitivity involves peripheral and central mechanisms. Increasing amounts of data support the argument that visceral hypersensitivity in IBS patients is tightly associated with neurotransmitters of the bowel. It has been demonstrated that neurochemical responses and visceral hyperalgesia are exaggerated via mechanisms involving $c$-fos and its receptors/transporters in the colon, mimicking IBS in neonatal MS stress-predisposed rats (Ren et al., 2007). However, our data from the same rat model suggest that SP and NK1R levels are not significantly altered in MS with or without repetitive CRD, although some minor decrease in these levels occurred. Based on these observations, we could conclude that maternal deprivation at an early life stage exerts miniscule effect on the expression and distribution of SP and its receptor along the brain-gut axis in neonatal MS rat model with visceral hypersensitivity. This suggests that SP and NK1R play a small role in the morphogenesis of visceral hyperalgesia in neonatal MS rat models. This appears to contrast with the aforementioned conclusion that SP and NK1R mediate visceral hypersensitivity. Several explanations may account for this discrepancy.

First, SP-containing nerve fibers are mainly distributed in the muscular layer of the gut (Faussone-Pellegrini, 2006), which may be either intrinsic or extrinsic. Chemical deletion of extrinsic afferent neurons did not alter the metallographical constituent of SP and neurokinin A (NKA) in the rat gut, while extrinsic neurons had a little role in the tachykininergic innervations of the muscle layers. This indicated that SP in the external muscle layer of the gut is mainly derived from intrinsic enteric neurons (Lecci et al., 2006). Therefore, it is difficult to determine the exact expression and distribution of SP in the extrinsic neurons in our experiment.

Second, IBS is a functional bowel disorder, and no specific biochemical or structural abnormalities can account for its mechanism, although SP plays an important part in distension-induced visceral pain, as is consistently supported by various reports. The role of SP or tachykinin NK1Rs in conveying the somatic pain response remains controversial in contrast with its chemically-induced visceral pain response. No difference in serum and colorectal mucosal SP levels was observed between IBS patients and healthy individuals, or between rectal hypersensitivity (+) and (-) IBS patients (Kerckhoffs et al., 2012). Laird et al. declared that the pain response was not altered in tachykinin NK1R knockout mice (Laird et al., 2000). In women with IBS, the transcription of transient receptor potential vanilloid 1 (TRPV1) and mucosal concentrations of SP were significantly higher, but the latter did not correlate with the severity of pain (Keszthelyi et al., 2013). The mechanisms of SP-mediated gastrointestinal function are complicated and contradictory. For example, SP can act as an endocrine, paracrine, or neuroendocrine factor.

Third, SP and NK1R mainly function in nociception associated with inflammation, which is supported by the notion that they are involved in dysregulated immune and inflammatory reactions in the intestinal mucosa of IBS patients (Weinstock, 2015). Neonatal MS rat model is a central neuromechanism-mediated model where neonatal mental stress transforms colonic functions in adult rats. No inflammation and/or infection were involved in the pathophysiology of visceral hypersensitivity in our study. MS-induced hyperalgesia could be mediated directly by inflammatory mediators released from mast cells, and thus no significant changes in SP and NK1R levels would occur at the nerve terminals.

Another possible explanation for this discrepancy is that NK1R signaling pathways do not control colonic motility and visceral hyperalgesia. Furthermore, Mule et al. showed

Genetics and Molecular Research 15 (3): gmr.15038999 
that an NK1R antagonist did not affected by a residual spontaneous colon motility, which was inhibited by several NK2R antagonists. This indicated that the predominant NK1R signaling pathways are not obviously associated with the modulation of residual colon motility and increased sensitivity of IBS rat colon to stress (Mulè et al., 2000). Meanwhile, based on experiments with mice lacking genes encoding for SP or NK1, or with NK1 antagonists, Laird et al. proposed the existence of two different hyperalgesia pathways, one of which was NK1 dependent and the other one did not require SP/NK1 signaling (Laird et al., 2000). The latter pathway may underpin colonic alteration in adult rats following neonatal maternal deprivation.

In addition, the developmental stage of rats in our experiment might be a notable factor, as the alterations that we focused on in this study were investigated in adult rats, and we did not assess young individuals. For instance, colonic mast cell hyperplasia is different at 4 or 12 weeks in maternally deprived rats, and mucosal nerve fiber density increases at 12 weeks of age but not at 4 weeks. This was assessed by the immunoreactivity of protein gene product 9.5 (PGP 9.5). At the same time, as assessed by calcitonin gene-related protein (CGRP) immunoreactivity, the fiber density remained constant compared with control rats (Barreau et al., 2008). Since DRN plays a vital role in visceral pain, down-regulation of pain control and up-regulation of pain modulation in the thalamus and the prefrontal cortex is important (Wang et al., 2009; Bravo et al., 2011) and consistent with reports that SP-containing neural pathways (including the hypothalamus) are significantly altered in a rat model of IBS with rectal distention (Carabotti et al., 2015). In contrast, our results revealed no significant differences in SP and NK1R levels between MS and NS treatment groups, before and after CRD. Thus, it is necessary to study the expression of SP and its receptor in the brain to further understand its distribution and to determine their roles in the morphogenesis of visceral hyperalgesia in neonatal MS rat models.

Taken together, our study investigated the expression and distribution of SP and NK1R along the brain-gut axis in neonatal MS rat. Using immunohistochemistry, we found no significant alterations in SP and NK1R levels in the colon, spinal cord, and the brainstem regardless of CRD. This suggested that neonatal MS might have little impact on SP and NK1R signal pathway in visceral afferent. Maternal deprivation, a successfully representative animal model for the study of IBS, should be used prudently and many experimental considerations should be taken into account when investigating specific neurotransmitters, such as SP and NK1R.

\section{Conflicts of interest}

The authors declare no conflict of interest.

\section{ACKNOWLEDGMENTS}

Research supported by a grant from the Experimental Animal Science and Technology Project of Zhejiang Province in China, \#2013C37032.

\section{REFERENCES}

Barreau F, Salvador-Cartier C, Houdeau E, Bueno L, et al. (2008). Long-term alterations of colonic nerve-mast cell interactions induced by neonatal maternal deprivation in rats. Gut 57: 582-590. http://dx.doi.org/10.1136/ gut.2007.126680

Genetics and Molecular Research 15 (3): gmr.15038999 
SP and its receptor in neonatal maternally separated rats

Boutaghou-Cherid H, Porcher C, Liberge M, Jule Y, et al. (2006). Expression of the neurokinin type 1 receptor in the human colon. Auton. Neurosci. 124: 9-17. http://dx.doi.org/10.1016/j.autneu.2005.10.002

Bravo JA, Dinan TG and Cryan JF (2011). Alterations in the central CRF system of two different rat models of comorbid depression and functional gastrointestinal disorders. Int. J. Neuropsychopharmacol. 14: 666-683. http://dx.doi. org $/ 10.1017 / \mathrm{S} 1461145710000994$

Bradesi S, Kokkotou E, Simeonidis S, Patierno S, et al. (2006). The role of neurokinin 1 receptors in the maintenance of visceral hyperalgesia induced by repeated stress in rats. Gastroenterology 130: 1729-1742. http://dx.doi. org/10.1053/j.gastro.2006.01.037

Carabotti M, Scirocco A, Maselli MA and Severi C (2015). The gut-brain axis: interactions between enteric microbiota, central and enteric nervous systems. Ann. Gastroenterol. 28: 203-209.

Chung EK, Zhang X, Li Z, Zhang H, et al. (2007). Neonatal maternal separation enhances central sensitivity to noxious colorectal distention in rat. Brain Res. 1153: 68-77.http://dx.doi.org/10.1016/j.brainres.2007.03.047

Coutinho SV, Plotsky PM, Sablad M, Miller JC, et al. (2002). Neonatal maternal separation alters stress-induced responses to viscerosomatic nociceptive stimuli in rat. Am. J. Physiol. Gastrointest. Liver Physiol. 282: G307-G316. http:// dx.doi.org/10.1152/ajpgi.00240.2001

Darmani NA, Wang Y, Abad J, Ray AP, et al. (2008). Utilization of the least shrew as a rapid and selective screening model for the antiemetic potential and brain penetration of substance P and NK1 receptor antagonists. Brain Res. 1214: 58-72. http://dx.doi.org/10.1016/j.brainres.2008.03.077

Faussone-Pellegrini MS (2006). Relationships between neurokinin receptor-expressing interstitial cells of Cajal and tachykininergic nerves in the gut. J. Cell. Mol. Med. 10: 20-32.http://dx.doi.org/10.1111/j.1582-4934.2006.tb00288.x

Inagaki S, Sakanaka M, Shiosaka S, Senba E, et al. (1982). Ontogeny of substance P-containing neuron system of the rat: immunohistochemical analysis--I. Forebrain and upper brain stem. Neuroscience 7: 251-277. http://dx.doi. org/10.1016/0306-4522(82)90165-8

Kerckhoffs AP, ter Linde JJ, Akkermans LM and Samsom M (2012). SERT and TPH-1 mRNA expression are reduced in irritable bowel syndrome patients regardless of visceral sensitivity state in large intestine. Am. J. Physiol. Gastrointest. Liver Physiol. 302: G1053-G1060.http://dx.doi.org/10.1152/ajpgi.00153.2011

Keszthelyi D, Troost FJ, Jonkers DM, Helyes Z, et al. (2013). Alterations in mucosal neuropeptides in patients with irritable bowel syndrome and ulcerative colitis in remission: a role in pain symptom generation? Eur. J. Pain 17: 1299-1306. http://dx.doi.org/10.1002/j.1532-2149.2013.00309.x

Kozlowski CM, Green A, Grundy D, Boissonade FM, et al. (2000). The 5-HT(3) receptor antagonist alosetron inhibits the colorectal distention induced depressor response and spinal c-fos expression in the anaesthetised rat. Gut 46: 474480. http://dx.doi.org/10.1136/gut.46.4.474

Kyloh M, Nicholas S, Zagorodnyuk VP, Brookes SJ, et al. (2011). Identification of the visceral pain pathway activated by noxious colorectal distension in mice. Front. Neurosci. 5: 16. http://dx.doi.org/10.3389/fnins.2011.00016

Laird JMA, Olivar T, Roza C, De Felipe C, et al. (2000). Deficits in visceral pain and hyperalgesia of mice with a disruption of the tachykinin NK1 receptor gene. Neuroscience 98: 345-352. http://dx.doi.org/10.1016/S0306-4522(00)00148-2

Lecci A, Capriati A, Altamura M and Maggi CA (2006). Tachykinins and tachykinin receptors in the gut, with special reference to NK2 receptors in human. Auton. Neurosci. 126-127: 232-249. http://dx.doi.org/10.1016/j. autneu.2006.02.014

Lu CL, Pasricha PJ, Hsieh JC, Lu RH, et al. (2005). Changes of the neuropeptides content and gene expression in spinal cord and dorsal root ganglion after noxious colorectal distension. Regul. Pept. 131: 66-73. http://dx.doi.org/10.1016/j. regpep.2005.06.008

Ma XP, Tan LY, Yang Y, Wu HG, et al. (2009). Effect of electro-acupuncture on substance P, its receptor and corticotropinreleasing hormone in rats with irritable bowel syndrome. World J. Gastroenterol. 15: 5211-5217. http://dx.doi. org/10.3748/wjg. 15.5211

Mulè F, D'Angelo S, Tabacchi G and Serio R (2000). Involvement of tachykinin NK2 receptors in the modulation of spontaneous motility in rat proximal colon. Neurogastroenterol. Motil. 12: 459-466. http://dx.doi.org/10.1046/ j.1365-2982.2000.00222.x

Ogawa M, Takamatsu M, Okamoto M, Baba H, et al. (2007). Iteration of high-frequency stimulation enhances long-lasting excitatory responses in the spinal dorsal horn of rats: characterization by optical imaging of signal propagation. Neurosci. Res. 57: 467-472. http://dx.doi.org/10.1016/j.neures.2006.11.011

O'Malley D, Dinan TG and Cryan JF (2011). Neonatal maternal separation in the rat impacts on the stress responsivity of central corticotropin-releasing factor receptors in adulthood. Psychopharmacology) 214: 221-229. http://dx.doi. org $/ 10.1007 / \mathrm{s} 00213-010-1885-9$

Renzi D, Pellegrini B, Tonelli F, Surrenti C, et al. (2000). Substance P (neurokinin-1) and neurokinin A (neurokinin-2) receptor gene and protein expression in the healthy and inflamed human intestine. Am. J. Pathol. 157: 1511-1522. http://dx.doi.org/10.1016/S0002-9440(10)64789-X

Genetics and Molecular Research 15 (3): gmr.15038999 
Ren TH, Wu J, Yew D, Ziea E, et al. (2007). Effects of neonatal maternal separation on neurochemical and sensory response to colonic distension in a rat model of irritable bowel syndrome. Am. J. Physiol. Gastrointest. Liver Physiol. 292: G849-G856.http://dx.doi.org/10.1152/ajpgi.00400.2006

Scheurer U, Drack E and Halter F (1994). Substance P activates rat colonic motility via excitatory and inhibitory neural pathways and direct action on muscles. J. Pharmacol. Exp. Ther. 271: 7-13.

Stam R, Ekkelenkamp K, Frankhuijzen AC, Bruijnzeel AW, et al. (2002). Long-lasting changes in central nervous system responsivity to colonic distention after stress in rats. Gastroenterology 123: 1216-1225. http://dx.doi.org/10.1053/ gast.2002.36029

Tjong YW, Ip SP, Lao L, Wu J, et al. (2011). Role of neuronal nitric oxide synthase in colonic distension-induced hyperalgesia in distal colon of neonatal maternal separated male rats. Neurogastroenterol. Motil. 23: 666-e278. http://dx.doi.org/10.1111/j.1365-2982.2011.01697.x

Wang GD, Wang XY, Liu S, Qu M, et al. (2014). Innervation of enteric mast cells by primary spinal afferents in guinea pig and human small intestine. Am. J. Physiol. Gastrointest. Liver Physiol. 307: G719-G731.http://dx.doi.org/10.1152/ ajpgi.00125.2014

Wang Z, Guo Y, Bradesi S, Labus JS, et al. (2009). Sex differences in functional brain activation during noxious visceral stimulation in rats. Pain 145: 120-128. http://dx.doi.org/10.1016/j.pain.2009.05.025

Weinstock JV (2015). Substance P and the regulation of inflammation in infections and inflammatory bowel disease. Acta Physiol. (Oxf.) 213: 453-461.http://dx.doi.org/10.1111/apha.12428

Yin JB, Wu HH, Dong YL, Zhang T, et al. (2014). Neurochemical properties of BDNF-containing neurons projecting to rostral ventromedial medulla in the ventrolateral periaqueductal gray. Front. Neural Circuits 8: 137. http://dx.doi. org/10.3389/fncir.2014.00137

Genetics and Molecular Research 15 (3): gmr.15038999 